\title{
Por que restringir testes psicológicos e para quem? Uma revisão narrativa
}

\author{
Why restrict psychological tests and for whom? A narrative review
}

Wesley de Mattos Machado'

Resumo: No Brasil, a área da Avaliação Psicológica atualmente discute sobre a decisão do Supremo Tribunal Federal de invalidar as restrições de venda dos testes psicológicos e o impacto dessa mudança é um campo a ser explorado pelos profissionais da Psicologia. A partir disso, este estudo tem os seguintes objetivos, investigar como a comercialização irrestrita de instrumentos psicológicos pode influenciar na Avaliação Psicológica, qual a necessidade de restringir a venda de testes e para quem aplicar essas restrições. A justificativa do estudo está na necessidade de discutir sobre a comercialização dos instrumentos psicológicos e sobre quais são os profissionais capacitados para aplicá-los, pois essa é uma discussão importante para a confiabilidade dos resultados na área da Avaliação Psicológica. O método deste estudo é uma revisão narrativa de materiais sobre as restrições de comercialização de testes psicológicos e dos acontecimentos que levaram até a decisão do Supremo Tribunal Federal. Com base na discussão apresentada, o presente estudo conclui, visando a qualidade dos resultados dos testes psicológicos, que há a necessidade das restrições de venda para público geral, mas que não se justifica proibir a venda dos testes para profissionais de outras áreas que tenham capacidade técnica e teórica para utilizá-los.

Palavras-chave: Avaliação psicológica; Comercialização de testes; Testes psicológicos.
Abstract: In Brazil, the Psychological Assessment nowadays discuss about the decision of the Brazilian Supreme Court to invalidate the restrictions sails of psychological tests, and the impact of this change is a field to be explored by the professionals of Psychology. From that, this study aims to investigate how the unrestricted commercialization of psychological instruments can influence the Psychological Assessment, what is the need to restrict the tests sails and for who to apply these restrictions. The justification of the study is the need to discuss about the psychological instruments commercialization and which are the prepared professionals to apply these instruments, because this is an important discussion for the reliability of results in the Psychological Assessment. The method of this study is a narrative review of the materials about the restrictions on the sales of psychological tests and of the events that led to the decision of the Supreme Court. Based on the discussion presented, this study concludes, aiming the quality of tests results, that there is the need of the restrictions sails for public, but it's not justified to restrict the sale of tests to professionals of other areas who have the technical and theoretical capacity to use them.

Keywords: Psychological assessment; Commercialization of tests; Psychological tests. 


\section{Introdução}

Atualmente, a área da Avaliação Psicológica discute sobre a decisão do Supremo Tribunal Federal de invalidar as restriçōes de venda dos testes psicológicos e 0 impacto dessa decisão é um campo a ser explorado pelos profissionais da área. Essa mudança não afeta a realização de um processo de avaliação psicológica, pois isto continua sendo função privativa do psicólogo, porém, a compra dos materiais usados para essas avaliações passa a ser liberada ao público geral. Por isso, discutir sobre a comercialização dos testes e sobre quem são os profissionais capacitados para aplicá-los é de grande importância para a confiabilidade dos resultados de uma avaliação psicológica, bem como discutir sobre os impactos da venda irrestrita desses instrumentos nas decisões tomadas a partir do seu uso. Portanto, este estudo tem como objetivos investigar como a livre comercialização de instrumentos psicológicos pode influenciar no uso dos testes na Avaliação Psicológica, qual a necessidade de restringir a venda e para quem. A justificativa deste estudo se baseia na necessidade de compreensão de quais podem ser as consequências da decisão do Supremo Tribunal Federal (STF) em avaliações psicológicas.

\section{Definições sobre avaliação psicológica}

Hutz, Bandeira eTrentini (2019) afirmam que a Avaliação Psicológica se configura como processo que visa conhecer e compreender o funcionamento psicológico e comportamental das pessoas. Esse processo avaliativo écapaz de orientar e influenciar decisões futuras em diversas áreas da Psicologia, como na investigação aprofundada sobre a pessoa e suas vivências em um processo de psicoterapia, na Psicologia do Trânsito como forma de avaliar a aptidão dos indivíduos para conduzir veículos ou no campo jurídico clarificando questões sobre as partes envolvidas, tanto de capacidade intelectual, quanto da possivel existência de psicopatologias. Esse grau de influência exige que a produção de dados desses processos seja a mais assertiva possivel. É com base nessa necessidade que a área da Avaliação Psicológica se estruturou como uma parte muito regulada pelo Conselho Federal de Psicologia (CFP) e por outras entidades da área de Avaliação.

No Brasil, o nascimento da Avaliação Psicológica se dá entrelaçado com o da Psicologia, pois antes mesmo da regulamentação da profissão já existiam laboratórios exercendo pesquisas sobre instrumentos psicológicos para avaliar indivíduos (Hutz, Bandeira \& Trentini, 2015). Em 1962, a lei federal de número 4.119, emitida pela Presidência da República (PR), instaura como funções privativas do psicólogo o diagnóstico psicológico, a orientação e seleção profissional, a orientação psicopedagógica e a solução de problemas de ajustamento. Desse modo, a Avaliação Psicológica passa a ser exclusiva do profissional formado em Psicologia (PR, 1962). Tal aspecto contribuiu para a segurança do trabalho dos profissionais da área, pois reduz as chances de erro nesse processo.

Quando se leva em conta que essa prática é restrita aos psicólogos, a Psicologia assume a função de ter o preparo necessário para realizar todo o processo, mas é importante salientar que mesmo sendo função exclusiva do psicólogo, a lguns autores questionam a qualidade dos profissionais acerca do uso de testes. Bandeira (2018) questiona se permitir que qualquer profissional formado em Psicologia faça uso dos testes pode influenciar na qualidade das avaliações psicológicas. A autora defende a necessidade de maior especialização nessa área de conhecimento, como forma de melhorar a atuação profissional. Questionar isso é um modo de buscar que esse processo tenha a qualidade que só o conhecimento aprofundado das técnicas usadas pode proporcionar, ou seja, é essencial que haja, além da formação em Psicologia, cursos de especialização sobre Avaliação Psicológica como requisito para um profissional aplicar tais instrumentos.

Ao ter especializações em Avaliação Psicológica reconhecidas e aprovadas pelo CFP, a Psicologia pode ter maiores ganhos no ensino sobre o uso dos testes e sobre os fundamentos necessários para atuar com avaliação. 0 ensino da Avaliação Psicológica exclusivamente nas graduações enfrenta problemas, como 0 ensino apenas dos procedimentos para a aplicação dos testes psicológicos e a pouca carga horária disponível na grade curricular para aprofundar em mais instrumentos (Bueno \& Peixoto, 2018). Esse tipo de ensino faz com que os alunos aprendam somente a aplicar os testes, de forma decorada e sem entender a fundo como os instrumentos funcionam ou como se dá 0 levantamento de informações a partir dos resultados, sem entender também como fazer a escolha do instrumento correto para cada ocasião.

Após reflexões como a de Bueno e Bandeira sobre a necessidade de especializações na área, o CFP lançou a Resolução número 18 de 2019, que torna a Avaliação Psicológica uma especialidade da Psicologia (CFP, 2019). A partir disso, os profissionais passam a poder realizar cursos de titulação de especialista na área ou em testes específicos, com as finalidades de aperfeiçoar 0 trabalho realizado com esses materiais, proporcionar um aprofundamento e um contato maior com as teorias e as características de cada instrumento estudado.

Na prática, o psicólogo tem na Avaliação Psicológica um importante meio de obter informações de forma detalhada e quantificada, sendo possível averiguar a assertividade de hipóteses que motivaram o processo avaliativo (Baptista et al., 2019). Hutz (2015) afirma que o processo de uma avaliação é um conjunto que engloba aspectos como a compreensão do funcionamento pessoal do avaliado e dos dados obtidos a partir das entrevistas, observaçōes, escalas e testes. Salienta também, que o processo da avaliação não se limita somente ao uso dos testes, mas 0 uso da testagem no campo da avaliação assumiu, no decorrer da história da área, o papel de dar cientificidade ao que está sendo apontado pelos profissionais.

A Avaliação Psicológica utiliza os testes e seus manuais para o levantamento de informaç̧ões e produção de dados científicos sobre os indivíduos avaliados (Primi, 2010). Baptista et al. (2019) diz que testes são uma forma padronizada de entender o funcionamento dos avaliados em aspectos emocionais e comportamentais. Os testes, ao explorar essas informaçōes, permitem que esses dados sejam colocados em um contexto e comparados com uma população geral, de forma a poder atribuir sentido aos dados obtidos. 0 teste de quociente de inteligência (Ql) é um exemplo disso, com base nas capacidades de solução de problemas, ele serve para mensurar características como raciocínio lógico e amplitude criativa, deste modo, calculando o quão inteligente é uma pessoa quando comparada com outras (Hutz et al., 2015). Essa nota de Ql sem um padrão de comparação seria apenas um número qualquer, mas com a padronização da população atendida pelo teste, passa a ter sentido e valor para a Avaliação Psicológica.

Primi (2018) diz que a necessidade de padronização e aumento da qualidade dos testes psicológicos impulsionou a criação do Sistema de Avaliação de Testes Psicológicos (SATEPSI), que tem como objetivo analisar os testes usados no Brasil e dar pareceres sobre a qualidade e a precisão deles, formando assim uma lista de instrumentos indicados para o uso. Algumas das contribuições do SATEPSI que mais influenciaram a prática do psicólogo 
foram a participação na elaboração e publicação das Resoluções 02/2003 (CFP, 2003), 05/2012 (CFP, 2012) e 09/2018 (CFP, 2018), que regulamentam 0 uso, a elaboração e a comercialização dos instrumentos psicológicos, que segundo o CFP (2003), por meio da Resolução 02/2003, tinham o resguardo da sua comercialização somente para psicólogos (Irigaray et al., 2020). A partir disso, com a criação do SATEPSI, das regulamentações e das restrições de comercialização dos instrumentos psicológicos, a Psicologia teve um ganho na qualidade dos testes e manuais usados no exercício da profissão, pois o CFP

Demonstrou, também, a aceitação das Editoras aos ditames da Resolução CFP nº 002/2003, de modo a permitir o controle adequado dos testes psicológicos, ressaltando que foi a partir da publicação da referida Resolução que começou a haver uma completa adequação dos testes psicológicos pelas Editoras (CFP, 2021).

\section{O processo judicial de inconstitucionalidade}

A partir de 2004, tramitou um processo contra as regulamentações sobre a comercialização restrita dos instrumentos psicológicos, essa ação judicial tratava sobre a inconstitucionalidade das resoluções do (FP. Neste ano, o Ministério Público (MP) da cidade de Uberaba (MG) entrou com um procedimento administrativo que tinha como objetivo esclarecer a razão das restrições do CFP para a venda de testes psicológicos. 0 CFP (2021) afirma que prestou todos os esclarecimentos requeridos na época. No mesmo ano, o MP de Uberaba requisitou que a Procuradoria Geral da República (PGR) instaurasse uma ação direta de inconstitucionalidade com a finalidade de invalidar as restrições de comercialização. Com base na requisição, no ano de 2005, a PGR pediu a abertura do processo junto ao STF, justificando que a restrição de comercialização violava a livre manifestação do pensamento e expressão da atividade intelectual dos autores e editoras, além de violar a difusão da cultura nacional e o livre acesso à informação para população em geral (STF, 2005).

Entretanto, com as editoras e autores aceitando as restrições impostas pelo CFP, fica claro que não há violação no que diz respeito a expressar sua atividade intelectual. Da forma que foram pensadas essas restrições, os trabalhos desses autores e editoras passaram a atingir somente pessoas aptas a fazerem uso dos instrumentos, minimizando os impactos negativos à sociedade. As restrições não têm o objetivo de privar 0 acesso à informação, mas sim de controlar e validar uma forma de avaliar aspectos mentais, comportamentais e de personalidade. As informações obtidas pelos testes necessitam de cuidados especializados por serem pessoais e sigilosas, tendo assim, que ter sua segurança, veracidade e resguardo garantidos pelo profissional aplicador dos instrumentos.

A partir do ano de 2005, o processo de inconstitucionalidade ficou paralisado, portanto, foram mantidas as restrições de comercialização até que fosse julgado por completo. No ano de 2018, com a posse do Ministro Alexandre de Moraes, o STF reativou o processo e deu início ao julgamento da questão. Em março de 2021, foi julgada inconstitucional a restrição da comercialização dos instrumentos psicológicos, sendo alegado, segundo o STF, que o diagnóstico psicológico é restrito ao Psicólogo, porém, os instrumentos psicológicos por serem de cunho científico, cultural e educativo, comparáveis a um livro de direito, não podem ser restritos aos profissionais de Psicologia, pois isto configura restrição do acesso universal à informação (STF, 2021).

As entidades reguladoras da Psicologia e Avaliação Psicológica se posicionaram contrárias à decisão do STF. 0 (FP (2021), acompanhado do Instituto Brasileiro de Avaliação Psicológica (IBAP), da Associação Brasileira de Rorschach e Métodos Projetivos (ASBRo), do Instituto Brasileiro de Neuropsicologia e Comportamento (IBNeC) e da Associação Brasileira de Psicologia de Tráfego (Abrapsit), pontua, em um pronunciamento em vídeo, que a livre comercialização dos testes e seus manuais afeta a capacidade da Psicologia de garantir qualidade nos resultados obtidos pelos instrumentos de Avaliação Psicológica. Alega também, que em nada se comparam os testes e seus manuais com livros didáticos de direito, pois a aquisição e leitura destes implica na capacidade direta de burlar um processo de avaliação psicológica, podendo assim, lesar a sociedade e a credibilidade da categoria de profissionais por resultados imprecisos e irreais.

0 Conselho Regional de Psicologia do Rio Grande do Sul (CRPRS, 2021), em uma nota emitida em seu site, acompanha o posicionamento do (FP, pois afirma que a decisão do STF implica necessariamente em um perigo para a veracidade dos resultados obtidos por meio de avaliações com instrumentos psicológicos. A entidade justifica sua posição alegando que a livre comercialização dos testes torna viável que pessoas comprem um instrumento e se preparem para um processo de avaliação psicológica, podendo dar respostas que escondam detalhes importantes e necessários para a composição do laudo desse processo.

Como pontuado pelas entidades reguladoras da Psicologia, há relação de influência da venda irrestrita de materiais psicológicos com os resultados obtidos nas avaliações. O STF erra ao propor que um livro de direito se assemelha em impacto social com um manual e um teste psicológico. 0 Ministro Alexandre de Moraes não leva em conta que as leis e suas interpretações presentes nos livros de Direito servem para ajudar no seu cumprimento ou no seu entendimento, enquanto a leitura de um manual ou o uso de um teste revela respostas e dados que fazem com que a pessoa, ao realizar uma avaliação psicológica, tenha capacidade de se planejar e se preparar para responder as perguntas e atividades de um teste, assim, podendo invalidar 0 instrumento utilizado.

\section{Restringir a comercialização de testes para quem?}

O CFP (2013) estabelece em uma cartilha sobre Avaliação Psicológica os deveres dos profissionais que conduzem as avaliações. No que se refere ao uso de testes psicológicos, o dever é de garantir a integridade dos instrumentos, evitando comercializá-los ou ensinar seus processos de aplicação e levantamento para pessoas não formadas em Psicologia. Ainda conforme o CFP, a pessoa que usar um instrumento psicológico deve estar preparada para isso, a instituição destaca que o preparo necessário para o uso correto desse material se dá a partir da combinação do respeito das normas dos manuais, da compreensão correta do que tratam estes e do respeito das condições estabelecidas para 0 uso ideal dos testes. Destaca também, que o profissional que usa um teste psicológico deve estar amplamente embasado em conhecimentos sobre seus referenciais teóricos, garantindo que a interpretação dos dados fornecidos pelos instrumentos utilizados seja a mais correta e assertiva possível.

Quando o CFP apresenta deveres como esses, a ideia é que a disseminação dos testes seja evitada para não invalidar resultados de futuras 
avaliações psicológicas, pois pessoas que não são profissionais preparados e que adquirem conhecimento dos métodos de testagem podem usar isso para burlar o processo de avaliação. Dando esse tipo de orientação para a classe da Psicologia, o CFP deixa claro que o profissional deve estar apto para manusear testes e seus manuais.

Em 2008, o Conselho Regional de Medicina do Estado de São Paulo (CREMESP) emitiu um parecer sobre as restrições de comercialização dos testes psicológicos. No documento a entidade ressalta que a atuação do Psicólogo sobre a Avaliação Psicológica está respaldada em lei, mas que a restrição da venda de testes e manuais excede os limites dela, pois configura, como afirmado pelo STF posteriormente, violação do acesso ao conhecimento científico e educacional. 0 médico que pediu o parecer do CREMESP afirma que há uma monopolização dos testes psicológicos pelos profissionais da Psicologia e que profissionais da Medicina que queiram usar os testes para outro fim que não as atribuições exclusivas dos Psicólogos, como pesquisa e diagnóstico psiquiátrico, não têm acesso aos instrumentos. O CREMESP salienta no parecer que os impedimentos de compra dos testes não têm respaldo na lei e que desde que o profissional faça uso dos testes para fins que não as atribuições do psicólogo, o uso e compra deve ser liberado (CREMESP, 2008).

Após o parecer do CREMESP, o CFP entrou em discussão com a área da Fonoaudiologia sobre a coleção NEUPSILIN, que engloba o Instrumento de Avaliação Neuropsicológica Breve e o Instrumento de Avaliação Neuropsicológica Breve Infantil. Em 2017, depois de debater com o Conselho Federal de Fonoaudiologia (CFFA) sobre o uso dessa coleção pelos profissionais Fonoaudiólogos, as duas entidades entraram em acordo e lançaram uma resolução conjunta que permite que tanto Psicólogos, quanto Fonoaudiólogos possam aplicar e corrigir os testes dessa coleção, desde que nos aspectos respectivos as suas áreas. A justificativa para a inclusão da Fonoaudiologia no grupo de profissionais aptos a usar esses instrumentos foi de que os testes da coleção NEUPSILIN abordam aspectos neuropsicológicos das duas áreas, e que os autores da coleção explicitaram que os testes podem ser usados por essas duas classes de profissionais (CFFA, 2017).

Ao levar em conta que o CFP e o CFFA entraram em acordo sobre o uso de um teste que era privativo da Psicologia, as alegações do médico e os esclarecimentos dados pelo CREMESP sobre as restrições de instrumentos que poderiam ser usados na Psiquiatria ganham força e justificativa. 0 profissional apresenta como questionamento o porquê de um médico não poder usar um instrumento de autoria de um colega de categoria (CREMESP, 2008). № site do SATEPSI (2021), é possível constatar que alguns testes privativos da Psicologia têm sua autoria compartilhada com profissionais da Medicina, como por exemplo, o Inventário de Depressão Beck (BDI-II), em que os autores são psicólogos e um psiquiatra.

A partir disso, a situação que se instaura sobre a questão é de se pensar qual a necessidade de restringir os instrumentos para profissionais de outras áreas, sendo que a autoria dos testes não é exclusiva da Psicologia e que atualmente existem distintas especializações direcionadas para testes específicos. A disponibilização de especializações voltadas aos testes abre espaço para que tanto os profissionais da Psicologia, quanto de outras áreas como a Psiquiatria, Fonoaudiologia ou Pedagogia, possam estar melhor preparados para usar um teste do que a atual exigência de ser graduado em Psicologia.

Diferentemente, quando uma pessoa sem conhecimento teórico faz 0 uso destes instrumentos na tentativa de se beneficiar, ela pode acabar se prejudicando, pois como pontuado, as avaliações psicológicas podem ser usadas para fins distintos, como a obtenção de uma carteira nacional de habilitação. Neste caso, esse processo serve como filtro para que pessoas a princípio inaptas para conduzir veículos sejam impedidas de obter essa permissão. Deste modo, quando uma pessoa burla uma situação como a do exemplo, ela pode não só estar colocando a própria vida em risco, mas também a de terceiros.

Além do risco direto contra a segurança e a vida, ainda existem outras variáveis prejudiciais com o uso incorreto dos testes, como diagnósticos feitos por leigos, ou o uso de testes como o de QI, já citado neste texto, que só tem validade quando posto em comparação correta com os dados certos e a partir de uma interpretação profissional. Logo, uma análise errada dos dados de um teste de QI pode, em algum grau, atribuir um diagnóstico de baixa capacidade intelectual, ou de capacidade elevada, dando noções falsas do real funcionamento intelectual da pessoa avaliada, guiando para direções erradas tratamentos psicológicos, psiquiátricos e a vida da pessoa como um todo.

Portanto, é importante que se mantenham as restrições sobre a venda de testes e manuais ao público geral, pois é uma forma de assegurar que esses instrumentos não tenham seu uso atribuído a problemas e danos sociais causados pelo despreparo de quem os utiliza. Entretanto, como diz Bandeira (2018), não é imprescindível que haja uma formação em Psicologia para fazer o uso de um teste psicológico, pois isso não assegura qualidade ao profissional.

\section{Método}

0 presente estudo se apresenta como uma revisão narrativa de cunho exploratório que faz uso de artigos, livros e materiais alternativos como vídeos, notas de posicionamento, leis e disposições dos conselhos e entidades reguladoras da Psicologia, Avaliação Psicológica, Fonoaudiologia e Medicina. As bases de dados Scielo, Pepsic e Google Acadêmico foram usadas e nelas foram elencadas as seguintes palavras-chave para a busca: Avaliação Psicológica, Comercialização de testes psicológicos e Uso de testes psicológicos. Foram usados como critério de inclusão artigos e livros em língua portuguesa publicados a partir de 2010, com o objetivo de analisar argumentos mais atualizados sobre o tema, a fim de produzir um estudo atual para discorrer sobre os fatos e teorias por trás da situação dos testes psicológicos.

Os critérios usados para inclusão de obras fizeram com que fossem selecionados quinze artigos e três livros, foram descartados dez artigos e o resto compôs o estudo. 0 descarte de tantos materiais se deu pela pouca relação com o tema do estudo, a escolha das obras e a leitura delas explicitou a falta de material científico sobre o tema. Deste modo, o estudo faz uso de bibliografia proveniente de materiais alternativos, tendo como finalidade ampliar a discussão e sua abrangência. A escolha desses materiais se deu por buscas nos sites dos conselhos regionais e federal de Psicologia, Fonoaudiologia e Medicina, por levantamento de leis e do histórico processual da ação movida no STF.

\section{Considerações finais}

0 presente estudo mostra, a partir das informações levantadas, que as restrições de comercialização impostas pelo Conselho Federal de Psicologia têm a finalidade de proteger os resultados fornecidos pelos processos de avaliação. Mostra também que apesar da decisão do Supremo Tribunal Federal se basear na lei de livre divulgação da cultura e expressão do pensamento, os autores dos testes se posicionaram de acordo com restringir a venda de suas obras 
e as próprias editoras que vendem os materiais também se mostraram em conformidade, evidenciando que não há lesados no que se refere a expressão intelectual.

Quanto a livre divulgação da cultura, cabe reflexão, pois como enfatizado pelos conselhos de Psicologia e pelas associações de Avaliação Psicológica, a venda irrestrita de testes pode implicar em perda de qualidade dos resultados de uma avaliação psicológica e como dito pelo CFP, os testes psicológicos tiveram melhor adequação depois que foram aplicadas as restrições em 2003.

Com base nisso, este estudo entende, em conformidade com as entidades reguladoras da Psicologia e da Avaliação Psicológica, que há a necessidade das restrições de venda dos instrumentos psicológicos, pois, ao facilitar que um processo avaliativo seja burlado, os resultados das avaliações podem vir a oferecer riscos para a saúde e segurança. Quando se assume que há risco nessas áreas da sociedade, entende-se que é menos danoso privar materiais psicológicos, do que dar brecha para que existam motoristas despreparados no trânsito ou pessoas erroneamente diagnosticadas no dia a dia dos serviços de saúde do país.

Porém, este estudo não concorda com as restrições no que se refere a não permitir a venda dos testes psicológicos para profissionais não cadastrados na rede de conselhos de Psicologia. Como por exemplo, os profissionais da Medicina que emitiram um parecer contra o impedimento da compra de testes psicológicos. A justificativa do CFP para liberar o uso de alguns instrumentos aos profissionais da Fonoaudiologia embasa os argumentos usados pelos profissionais da Medicina, pois, se o CFP entende que o NEUPSILIN aborda temas interdisciplinares com a Fonoaudiologia, não é necessário ir muito longe para perceber que vários temas da Psicologia são interdisciplinares com a Psiquiatria, inclusive, alguns instrumentos são obras de profissionais dessa área.

Se um profissional da Medicina tem condições de criar e validar um teste sob as normas do Conselho Federal de Psicologia, entende-se que aos demais profissionais da categoria cabe a possibilidade de fazer o uso desses instrumentos, desde que em suas respectivas funções e com o devido preparo fornecido pelas especializações disponíveis para os testes.

Como levantado por Bandeira (2018), e discutido neste estudo, o que interfere para maior qualidade dos resultados obtidos pelos testes não é a formação em Psicologia, pois o graduando nem sempre tem o contato ideal com os instrumentos psicológicos, mas sim o preparo do profissional que vai utilizá-los, o entendimento sobre a teoria por trás do instrumento e a compreensão das regras de utilização. Sendo assim, a partir do momento que a área da Avaliação Psicológica passou a ter títulos de especialização e cursos de preparo para a aplicação de testes específicos, todo profissional que tenha a necessidade de usar um instrumento psicológico pode se tornar apto a fazê-lo. Então não há justificativa para privar profissionais que tenham uma especialização, preparo e entendimento sobre um teste, de adquiri-lo e usá-lo de forma correta e com a finalidade de adicionar qualidade e precisão às atuações nas suas áreas.

No decorrer da pesquisa, notou-se a escassa disponibilidade de materiais teóricos que discutam diretamente sobre os impactos da venda irrestrita dos testes psicológicos. Essa escassez de materiais dificulta a execução de estudos sobre a temática e deixa brecha para que outras áreas de conhecimento, como a do Direito, assumam que essas resoluções têm o objetivo de restringir mercado e privar conhecimentos, e que passem a questionar a validade de tais resoluções e sua aplicabilidade no contexto social. Recomenda-se, com base nessa escassez, que o tema seja melhor explorado e aprofundado para que possa haver uma maior compreensão sobre ele e um debate mais rico sobre para quem os testes devem ser restritos e os motivos dessas restrições.

\section{Referências}

Bandeira, D. (2018). A controvérsia do uso dos testes psicológicos por psicólogos e não psicólogos. Psicologia: (iência e Profissão, 38(spe). doi: https:// doi.org/10.1590/1982-3703000208860

Baptista, M. N., Nascimento, M. M., Reppold, C.T., Nunes, C. H. S. S., Carvalho, L. F. Primi, R., ... Pasquali, L. (Orgs.). (2019). Compêndio de avaliação psicológica. Petrópolis, RJ: Vozes.

Bueno, J. M. H., \& Peixoto, E. M. (2018). Avaliação psicológica no Brasil e no mundo. Psicologia: Ciência e Profissão, 38(spe). doi: https://doi. org/10.1590/1982-3703000208878

Conselho Federal de Fonoaudiologia. (2017). Resolução CFFA nº 001/2017: Institui conjuntamente regras para a utilização da Coleção NEUPSILIN para uso exclusivo dos/as Fonoaudiólogos/as e Psicólogos/as. Recuperado de: https://www.fonoaudiologia.org.br/resolucoes/resolucoes_html/ CFFa_N_001_17.htm

Conselho Federal de Psicologia. (2003). Resolução CFP nº 002/2003: Definee regulamenta 0 uso, a elaboração e a comercialização de testes psicológicos e revoga a Resolução CFP nº 025/2001. Recuperado de: https://site.cfp.org. br/wp-content/uploads/2003/03/resoluca02003_02_Anexo.pdf Conselho Federal de Psicologia. (2012). Resolução CFP nº 005/2012: Altera a Resolução CFP n. ${ }^{0}$ 002/2003, que define e regulamenta o uso, a elaboração e a comercialização de testes psicológicos. Recuperado de: https://site.ffp. org.br/wp-content/uploads/2012/03/Resolucao_CFP_005_12_1.pdf Conselho Federal de Psicologia. (2018). Resolução CFP nº 009/2018: Estabelece diretrizes para a realização de Avaliação Psicológica no exercício profissional da psicóloga e do psicólogo, regulamenta o Sistema de Avaliação de Testes Psicológicos - SATEPSI e revoga as Resoluções no 002/2003, no 006/2004 e no 005/2012 e Notas Técnicas no 01/2017 e 02/2017. Recuperado de: https://atosoficiais.com.br/lei/avaliacao-psicologica-cfp?origin=instituicao

Conselho Federal de Psicologia. (2019). Resolução CFP nº 18/2019: Reconhece a Avaliação Psicológica como especialidade da Psicologia e altera a Resolução CFP no 13, de 14 de setembro de 2007, que institui a Consolidação das Resoluções relativas ao Título Profissional de Especialista em Psicologia. Recuperado de: https://atosoficiais.com.br/cfp/resolucao-do-exercicio-profissional-n-18-2019-reconhece-a-avaliacao-psicologica-como-especialidade-da-psicologia-e-altera-a-resolucao-cfp-no-13-de-14 -de-setembro-de-2007-que-institui-a-consolidacao-das-resolucoes-relativas-a0-titulo-profissional-de-especialista-em-psicologia?origin=instituicao\&utm_source =nota

Conselho Federal de Psicologia. (2021). Decisão do STF sobre testes psicológicos: perguntas e respostas sobre os impactos da decisão do Supremo Tribunal Federal e estratégias de atuação do CFP em defesa da categoria. Recuperado de: https://site.cfp.org.br/perguntas-e-respostas-decisao-do-stf-sobre-testes-psicologicos/

Conselho Federal de Psicologia. (2021). Teste Psicológico: (FP realiza pronunciamento sobre decisão do Supremo Tribunal Federal [Vídeo transmissão]. Recuperado de: https://site.cfp.org.br/teste-psicologico-cfp-realiza-pronunciamento-sobre-decisao-do-supremo-tribunal-federal/ 
Conselho Regional de Medicina do Estado de São Paulo. (2008). Parecer nº 61981: Sobre a Resolução do Conselho Federal de Psicologia no. 02/2003 que restringe a utilização e comercialização de testes psicológicos apenas aos profissionais da psicologia. Recuperado de: http://www.cremesp. org.br/?siteAcao $=$ Pareceres\&dif=s\&ficha $=1 \& i d=7706 \&$ tipo $=$ PARE CER\&orgao=Conselho\%20Regional\%20de\%20Medicina\%20do\%20 Estad0\%20de\%20S\%E30\%20Paulo\&numero=61981\&situacao $=\& d a-$ $\mathrm{ta}_{\mathrm{a}}=11-01-2008$

Conselho Regional de Psicologia do Rio Grande do Sul. (2021). Nota de Posicionamento - Ação Direta de Inconstitucionalidade no 3481. CRPRS. Recuperado de: https://www.crprs.org.br/noticias/nota-de-posicionamento---acao-direta-de-inconstitucionalidade-n-3481

Hutz, C. S., Bandeira, D. R., \& Trentini, C. M. (Orgs.) (2015). Psicometria. Porto Alegre, RS: ARTMED.

Irigaray, T. Q., Schutz, D. M., Farina, M., Yates, M., Lima, M. P., Gonzatti, V. (Orgs.) (2020). Avaliação psicológica no contexto contemporâneo. Porto Alegre, RS: EDIPUCRS.

Presidência da República, Casa Civil. (1962). Leino 4.119, de 27 de agosto de 1962. Recuperado de: http://www.planalto.gov.br/ccivil_03/leis/19501969//4119.htm\#: : :text=Disp\%C3\%B5e\%20s\%C3\%B4bre\%200s\%20 cursos\%20de,regulamenta\%20a\%20profiss\%(3\%A30\%20de\%20psic\%C3\%B3logo.\&text=Art.,de\%20bacharelado\%2C\%20licenciado\%20 e\%20Psic\%C3\%B3logo

Primi, R. (2010). Avaliação psicológica no Brasil: Fundamentos, situação atual e direções para o futuro. Psicologia: Teoriae Pesquisa, 26(spe). doi: https:// doi.org/10.1590/S0102-37722010000500003

Primi, R. (2018). Avaliação psicológica no século XXI: De onde viemos e para onde vamos. Psicologia: Ciência e Profissão, 38(spe). doi: https://doi. org/10.1590/1982-3703000209814

Sistema de Avaliação de Testes Psicológicos. (2021). Testes favoráveis. Recuperado de: https://satepsi.cfp.org.br/testesFavoraveis.cfm

Supremo Tribunal Federal. (2005). PGR contesta resolução do Conselho Federal de Psicologia. Portal de Notícias do Supremo Tribunal Federal. Recuperado de: http://portal.stf.jus.br/noticias/verNoticiaDetalhe. asp?idConteudo $=64711 \&$ ori $=1$

Supremo Tribunal Federal. (2021). STF veda limitação de acesso a testes psicológicos a profissionais habilitados. Portal de Notíías do Supremo Tribunal Federal. Recuperado de: https://portal.stf.jus.br/noticias/verNoticiaDetalhe.asp?idConteudo=462049\&ori=1 